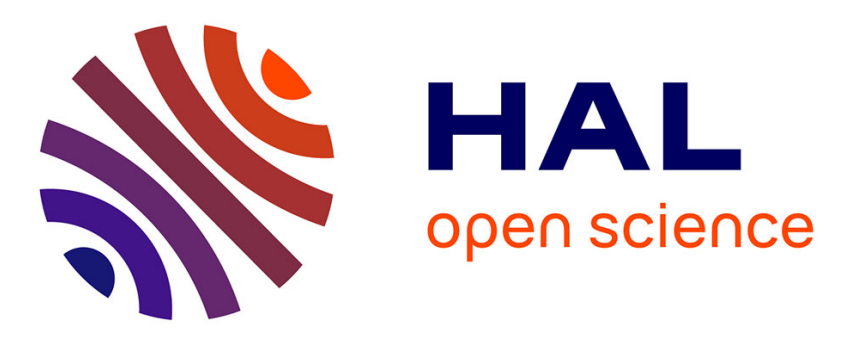

\title{
Étude de l'ordre magnétique sous pression dans CrTe par diffraction neutronique
}

\author{
B. Lambert-Andron, N.P. Grazhdankina, C. Vettier
}

\section{To cite this version:}

B. Lambert-Andron, N.P. Grazhdankina, C. Vettier. Étude de l'ordre magnétique sous pression dans CrTe par diffraction neutronique. Journal de Physique Lettres, 1978, 39 (3), pp.43-45. 10.1051/jphyslet:0197800390304300 . jpa-00231438

\section{HAL Id: jpa-00231438 https://hal.science/jpa-00231438}

Submitted on 1 Jan 1978

HAL is a multi-disciplinary open access archive for the deposit and dissemination of scientific research documents, whether they are published or not. The documents may come from teaching and research institutions in France or abroad, or from public or private research centers.
L'archive ouverte pluridisciplinaire HAL, est destinée au dépôt et à la diffusion de documents scientifiques de niveau recherche, publiés ou non, émanant des établissements d'enseignement et de recherche français ou étrangers, des laboratoires publics ou privés. 


\title{
ÉTUDE DE L'ORDRE MAGNÉTIQUE SOUS PRESSION DANS CrTe PAR DIFFRACTION NEUTRONIQUE
}

\author{
B. LAMBERT-ANDRON
}

Institut Laue Langevin, BP 156 X, 38042 Grenoble Cedex, France

\author{
N. P. GRAZHDANKINA
}

Institut de Physique des Métaux, Sverdlovsk, U.R.S.S.

\author{
C. VETTIER
}

Laboratoire de Magnétisme, C.N.R.S., av. des Martyrs, 38042 Grenoble, France

(Reçu le 14 novembre 1977, accepté le 8 décembre 1977)

\begin{abstract}
Résumé. - Le composé ferromagnétique CrTe a été étudié par diffraction neutronique dans une cellule haute pression afin de connaître l'évolution de l'ordre magnétique en fonction de la température et de la pression. Des mesures de résonance électronique ont montré la disparition du ferromagnétisme au-dessus de $P_{\mathrm{c}}=28 \mathrm{kbar}$. Les expériences de diffraction neutronique ont confirmé cette disparition à $P>28 \mathrm{kbar}$ pour $T=100 \mathrm{~K} ; 4,2 \mathrm{~K}$ et montré qu'il n'y a pas de nouveau mode magnétique. La faible composante antiferromagnétique persiste avec le même ordre de grandeur.

Abstract. - The magnetic compound CrTe was studied by neutron diffraction in a high pressure cell in order to evaluate the magnetic ordering as a function of temperature and pressure. E.S.R. measurements showed that ferromagnetism vanishes above $P_{\mathrm{c}}=28 \mathrm{kbar}$. The neutron diffraction experiments confirmed this disappearance at $P>28 \mathrm{kbar} ; T=100 \mathrm{~K} ; 4.2 \mathrm{~K}$ and showed that no new magnetic mode appears. The weak antiferromagnetic component persists with the same order of magnitude.
\end{abstract}

1. Introduction. - L'étude des structures magnétiques des solutions solides $\mathrm{CrTe}-\mathrm{CrSb}$ [1] a mis en évidence un grand nombre d'arrangements de spins : structures ferromagnétiques, antiferromagnétiques ou obliques. En particulier, CrTe est un composé ferromagnétique de structure hexagonale du type NiAs [2], $T_{\mathrm{c}}=343 \mathrm{~K}$, l'axe de facile aimantation est parallèle à l'axe c. A basse température une faible composante antiferromagnétique a été observée par plusieurs auteurs [1].

Les interactions magnétoélastiques sont fortes :

$$
\frac{\mathrm{d} T_{\mathrm{c}}}{\mathrm{d} P}=-6 \mathrm{~K} / \mathrm{kbar}[3]
$$

et la magnétostriction spontanée est importante [4].
L'application d'une pression hydrostatique se révèle donc être un moyen utile pour l'étude de la stabilité des différentes structures (ceci malgré la faible compressibilité $\simeq 1,4 \times 10^{-3} / \mathrm{kbar}$ [5]). Les mesures de résonance électronique faites par Shanditsev et al. [6] ont montré que le ferromagnétisme disparaît au-dessus de 28 kbar (Fig. 1).

Ces expériences ne permettant pas de voir s'il existe un mode antiferromagnétique, la question se pose de savoir si une nouvelle phase magnétique apparaît au-dessus de $P_{\mathrm{c}}$ ou si le composé devient paramagnétique.

Nous avons donc entrepris l'étude du diagramme de phase $P-T$, par diffraction neutronique, pour préciser le comportement magnétique de $\mathrm{CrTe}$ à haute pression et basse température. La connaissance de ce comporte- 


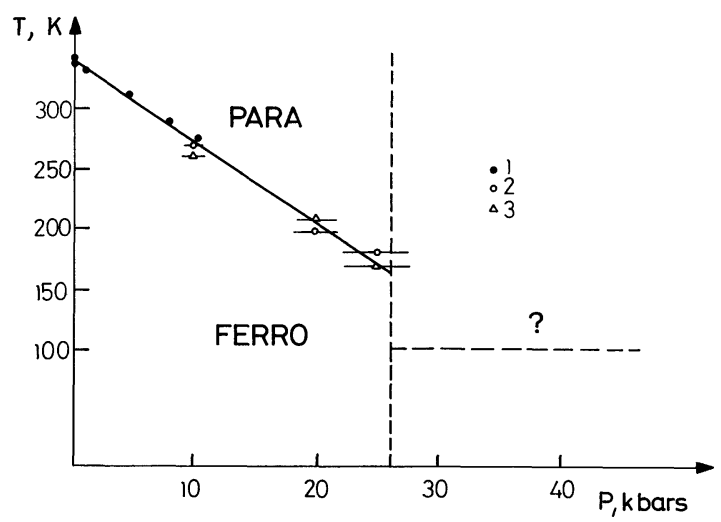

Fig. 1. - Variation de $T_{\mathrm{c}}$ en fonction de la pression : 1) pression hydrostatique ; 2,3 ) pression quasihydrostatique (différentes expériences). La ligne pointillée verticale définit la limite d'existence de la phase ferromagnétique; la ligne pointillée horizontale est la limite de température inférieure atteinte dans les expériences.

[Pressure dependence of the Curie point $T_{\mathrm{c}}$ of chromium telluride : 1) hydrostatic pressure ; 2, 3) quasi-hydrostatic pressure (different experiments). The vertical dashed line defines the limit of existence of the ferromagnetic phase; the horizontal dashed line is the lower limit reached in our experiment.]

ment est d'un grand intérêt pour étudier l'influence de la variation des distances interatomiques sur les mécanismes de superéchange et d'échange direct.

2. Expériences par diffraction neutronique. - Les mesures ont été faites à l'Institut Laue Langevin sur un spectromètre à 2 axes $\mathrm{D} 2$ avec une longueur d'onde $\lambda=1,29 \AA$. La cellule de pression est constituée d'un cylindre d'Alumine [7]. La pression est appliquée par deux pistons qui compriment l'échantillon auquel on a ajouté une faible quantité de $\mathrm{NaCl}$ pour déterminer la pression à l'intérieur de la cellule à partir de l'équation d'état de $\mathrm{NaCl}$ [8]. La cellule de pression est montée dans un cryostat conventionnel. On a pu ainsi obtenir des diagrammes avec :

$$
\begin{aligned}
& P \simeq 1 \mathrm{kbar}, 15 \mathrm{kbar}, 35 \mathrm{kbar}, \\
& T \simeq 9 \mathrm{~K}, 100 \mathrm{~K}, 300 \mathrm{~K} .
\end{aligned}
$$

Outre le spectre de CrTe dont la quantité est faible $<1 \mathrm{~g}$, les diagrammes obtenus comportent le spectre de l'alumine de la cellule et celui de $\mathrm{NaCl}$. Le bruit de fond dû à la cellule de pression est élevé ( $\simeq 500$ coups/min.). D'autre part, l'intensité observée d'une raie du composé est de l'ordre de 800 coups/min., on ne peut donc espérer une étude quantitative de la grandeur et de l'orientation des moments magnétiques. Cette difficulté pourrait être levée en utilisant des cellules H.P. à angles de diffusion fixes par des méthodes de temps de vol [9] ou en utilisant un spectromètre à 3 axes [10]. Mais ces cellules ne sont pas utilisables à basse température.

Une étude complémentaire a été effectuée au C.E.N. Grenoble, à pression atmosphérique, sans cellule, pour avoir un diagramme de référence du composé seul : à $T=4,2 \mathrm{~K}$ on constate l'existence du ferromagnétisme et d'une faible composante antiferromagnétique. Sous pression on obtient les résultats suivants :

Pour $P \simeq 15$ kbar.

TABLEAU I

Résultats de diffraction neutronique $\grave{a} P=15 \mathrm{kbar} \pm 2 \mathrm{kbar}$

[Results of neutron diffraction

\begin{tabular}{|c|c|c|}
\hline$T$ & $h k l$ & Intensités \\
\hline - & - & - \\
\hline $298 \mathrm{~K}$ & $\begin{array}{l}{[100]} \\
\quad+\text { contribution } \\
\text { de } \mathrm{Al}_{2} \mathrm{O}_{3}\end{array}$ & $13156 \pm 400$ \\
\hline $7 \mathrm{~K}$ & $\begin{array}{l}{[100]} \\
\quad+\text { contribution } \\
\text { de } \mathrm{Al}_{2} \mathrm{O}_{3}\end{array}$ & $20903 \pm 500$ \\
\hline $7 \mathrm{~K}$ & $\begin{array}{l}\text { [101] } \\
\text { (Indices donnés } \\
\text { dans la maille } \\
\text { antiferromagnéti- } \\
\text { que) }\end{array}$ & $1250 \pm 430$ \\
\hline
\end{tabular}
$P=15 \mathrm{kbar} \pm 2 \mathrm{kbar}]$

Après la correction due à l'agitation thermique on trouve :

Intensité de [100] ferromagnétique $\quad=7200 \pm 900$. Intensité de [101] antiferromagnétique $=1250 \pm 430$.

Pour $P \simeq 35$ kbar.

\section{TABLEAU II}

Résultats de diffraction neutronique $\grave{a} P=35 \mathrm{kbar} \pm 3 \mathrm{kbar}$

[Results of neutron diffraction

\begin{tabular}{|c|c|c|}
\hline$T$ & - $h k l$ & Intensités \\
\hline - & - & - \\
\hline $298 \mathrm{~K}$ & $\begin{array}{l}{[100]} \\
\quad+\text { contribution } \\
\text { de } \mathrm{Al}_{2} \mathrm{O}_{3}\end{array}$ & $14817 \pm 400$ \\
\hline $100 \mathrm{~K}$ & $\begin{array}{l}{[100]} \\
\quad+\text { contribution } \\
\text { de } \mathrm{Al}_{2} \mathrm{O}_{3}\end{array}$ & $15849 \pm 400$ \\
\hline $9 \mathrm{~K}$ & $\begin{array}{l}{[100]} \\
\quad+\text { contribution } \\
\text { de } \mathrm{Al}_{2} \mathrm{O}_{3}\end{array}$ & $17000 \pm 630$ \\
\hline $9 \mathrm{~K}$ & $\begin{array}{l}{[101]} \\
\text { (Indices donnés } \\
\text { dans la maille } \\
\text { antiferromagnéti- } \\
\text { que) }\end{array}$ & 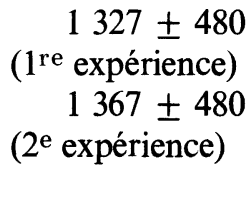 \\
\hline
\end{tabular}
$P=35 \mathrm{kbar} \pm 3 \mathrm{kbar}]$ 
Après la correction d'agitation thermique et dans la limite des barres d'erreur on trouve une contribution ferromagnétique nulle pour $T \simeq 100 \mathrm{~K}, 9 \mathrm{~K}$.

Nous avons fait une expérience à $T=100 \mathrm{~K}$, cette température étant la plus basse atteinte dans les mesures E.S.R. de Shanditsev [6]. La disparition du ferromagnétisme pour $P>P_{\mathrm{c}}$ est confirmée par les résultats de diffraction neutronique, mais on constate le maintien de la raie antiferromagnétique avec le même ordre de grandeur qu'à $P<P_{\mathrm{c}}$. L'intensité de [101] antiferromagnétique $\simeq 1345 \pm 480$. Les conditions expérimentales ne permettent pas de faire une comparaison quantitative de la composante antiferromagnétique à $P \simeq 15 \mathrm{kbar}$ et $P \simeq 35 \mathrm{kbar}$.

3. Discussion. - Les expériences de diffraction neutronique apportent les résultats nouveaux suivants :

1) A basse température et $P \simeq 35 \mathrm{kbar}$, le ferromagnétisme a disparu effectivement.

2) Il n'apparaît pas à $P \simeq 35 \mathrm{kbar}$, dans les limites de sensibilité de l'expérience, un mode magnétique nouveau.

3) La faible composante antiferromagnétique observée à pression ambiante par plusieurs auteurs [1] et nous-même, existe toujours à $P \simeq 35 \mathrm{kbar}$ avec le même ordre de grandeur.
La discontinuité de $T_{\mathrm{c}}$ en fonction de $P$ sans apparition d'une nouvelle phase antiferromagnétique nous permet d'écarter toute analyse du type Bean-Rodbell [11]. Selon cette théorie thermodynamique, l'application d'une pression extérieure peut altérer la nature de la transition magnétique d'une substance qui présente une forte dépendance des intégrales d'échange avec les distances interatomiques. On peut alors attendre, comme l'envisageait Shanditsev [6], l'apparition d'antiferromagnétisme. La persistance de la faible composante antiferromagnétique déjà observée à basse pression montre que le magnétisme ne disparaît pas à haute pression jusqu'à $P \simeq 35 \mathrm{kbar}$, et que l'échange direct $\mathrm{Cr}-\mathrm{Cr}$ donnant lieu à une interaction négative existe toujours.

Notons qu'un des grands intérêts de ce composé est le caractère de ses électrons $3 \mathrm{~d}$. En effet CrTe n'est pas un composé purement ionique, il présente des propriétés de conductibilité métallique et l'ordre de grandeur des moments magnétiques observés est inférieur à ceux d'un corps ionique. Ceci met en évidence une délocalisation partielle des électrons $3 \mathrm{~d}$ du chrome. Des études ultérieures par diffraction neutronique sur monocristaux en particulier devraient permettre de préciser le caractère collectif ou localisé des électrons 3d dans $\mathrm{CrTe}$.

\section{Bibliographie}

[1] Cox, D. E., Shirane, G., Takei, W. J., Proceedings of the Int. Conf. Magnetism, Nottingham 1964, p. 291.

TakeI, W. J., Cox, D. E., Shirane, G., J. Appl. Phys. 37 (1966) 973.

WinTENBERGER, M., MAKovetSKy, M., non publié.

[2] Chevreton, M., Bertaut, E. F., JellineK, F., Acta Crystallogr. 16 (1963) 431.

[3] Ido, H., Kaneko, T., Kamigaki, K., J. Phys. Soc. Japan 22 (1967) 1418.

Galkin, A. A., ZavadskiI, Z. A., Sinelnikov, B. Ya., Sov. Phys. Solid State 14 (1972) 125.

Grazhdankina, N. P., Gaidurov, L. G., Rodisnov, K. P., OleiniK, M. I., ShChipanov, V. A., Sov. Phys. JETP 13 (1961) 297.

[4] Gordienko, V. A., Zubenko, V. V., Nikolaev, V. I., Sov. Phys. JETP 30 (1970) 864.
Burkhanov, A. M., Fakidov, I. G., Sov. Phys. Solid State 15 (1974) 1404.

[5] Nagasaki, H., Wakabayashi, I., Minomura, S., J. Phys. Chem. Solids 30 (1969) 2405.

[6] Shanditsev, V. A., Vereskchagin, L. F., Yakovlev, E. N., Grazhdankina, N. P., Aleeva, T. I., Sov. Phys. Solid State 15 (1973) 146.

[7] Bloch, D., Paureau, J., Voiron, J., Parisot, G., Rev. Sci. Instrum. 47 (1976) 296.

[8] DeCKer, D. L., J. Appl. Phys. 42 (1971) 3239.

[9] Brugger, R. M., Bennion, R. B., Worlton, T. G., Myers, W. R., Trans. Am. Crystallogr. Assoc. 5 (1969) 141.

[10] Buras, B., Kofoed, W., LebeCh, B., BäCKSTRÖM, G., Ris $\varnothing$ Report 357, Research Establishment Ris $\varnothing$, Denmark.

[11] Bean, C. P., Rodbell, D. S., Phys. Rev. 126 (1962) 104. 